\title{
Detection of Neutralizing Antibody Against Japanese Encephalitis Virus in Wild Boars of Korea
}

\author{
Dong-Kun Yang ${ }^{1 *}$, Ha-Hyun Kim ${ }^{1}$, Bang-Hun Hyun ${ }^{1}$, Seong-In Lim ${ }^{1}$, \\ Yun-Kyoung Nam $^{2}$, Jin-Ju Nah ${ }^{1}$ and Jae-Young Song ${ }^{1}$ \\ ${ }^{I}$ Animal, Plant and Fishery Quarantine Inspection Agency, MIFAFF, Anyang; ${ }^{2}$ College of Veterinary Medicine, JeonBuk \\ National University, JeonJu, Korea
}

\begin{abstract}
Several species of animals, including horses and pigs, can be infected with Japanese encephalitis virus (JEV). Wild boars (Sus scrofa) are also considered to be an effective amplifying host for JEV in wild environments. In this study, 288 blood samples were collected from wild boars in eight Korean provinces, and antibodies against JEV were detected using a virus neutralizing assay. The results showed that $66.0 \%$ (190/288) of wild boars in Korea had neutralizing antibodies against JEV. We found no significant differences in the seroprevalence of JEV among provinces $(p>0.05)$. The results indicate that wild boars in Korea have been exposed to JEV, suggesting that these boars may play an important role in amplifying and carrying JEV to other regions of Korea. The result of this study may be helpful for planning preventive measures.
\end{abstract}

Key Words: Japanese encephalitis virus, Virus neutralization, Wild boar

More than 67,000 cases of Japanese encephalitis (JE) are reported to the World Health Organization annually, and the overall human incidence rate corresponds to 1.8 per 100,000 (1). Less than $1 \%$ of human JE cases show typical symptoms, such as coma and death. Survivors have significant neurologic sequelae (2). Japanese encephalitis virus (JEV) is a mosquito-borne virus that causes encephalitis in humans and horses and reproductive failure in pregnant sows. JEV has been recently recognized as a re-emerging pathogen because it has expanded into new territories, such as the Torres Strait and Australia (2 4). In addition, climate changes caused by recent global warming events and extreme weather may have significantly impacted the transmission of vector-borne diseases such as JEV, West
Nile virus (WNV), and tick-borne encephalitis virus (TBEV). Rapid weather and climate changes can directly or indirectly affect the activity of amplifying hosts and mosquitoes. According to data obtained from the Korean Center for Disease Control and Prevention, 45 cases of JEV were reported between 2007 and 2010, and more than half of the JEV cases occurred in 2010 (5).

JEV-infected mosquitoes bite several species of animals, including horses, wild birds, and pigs. Horses are known as dead-end hosts, and infection sometimes causes fatal illness; however, other animals bitten by mosquitoes show subclinical symptoms $(6,7)$. Pigs and wild birds, such as the heron and egret, are considered to be amplifying hosts because they produce high titers of JEV in the blood. In

\footnotetext{
Received: September 10, 2012/ Revised: November 14, 2012/ Accepted: November 19, 2012

* Corresponding author: Dong-Kun Yang. Animal, Plant and Fishery Quarantine Inspection Agency, 175 Anyang-ro, Anyang-si, Gyeonggi-do 430-757, Korea. Phone: +82-31-467-1783, Fax: +82-31-467-1797, e-mail: yangdk@korea.kr

${ }^{* *}$ This work was supported financially by a grant (B-AD14-2010-11-03) from the Animal, Plant and Fisheries Quarantine and Inspection Agency, Ministry for Food, Agriculture, Forestry and Fisheries, Korea.

(c) This is an Open Access article distributed under the terms of the Creative Commons Attribution Non-Commercial License (http://creativecommons.org/license/by-nc/3.0/).
} 
Table 1. Regional distribution of JEV antibodies from Korean wild boars

\begin{tabular}{cccccccccc}
\hline \hline & \multicolumn{10}{c}{ Provinces } \\
\cline { 2 - 8 } JEV & GW $^{\mathrm{a}}$ & GG & GN & GB & JN & JB & CN & CB & Total \\
\hline \multirow{2}{*}{ Positive rate (\%) } & $\mathbf{2 7 / 5 2}$ & $\mathbf{4 5 / 5 5}$ & $\mathbf{2 1 / 3 2}$ & $\mathbf{2 1 / 3 4}$ & $\mathbf{3 0 / 4 7}$ & $\mathbf{3 / 4}$ & $\mathbf{2 7 / 3 9}$ & $\mathbf{1 6 / 2 5}$ & $\mathbf{1 9 0 / 2 8 8}$ \\
& $\mathbf{( 5 1 . 9 )}$ & $\mathbf{( 8 1 . 8 )}$ & $\mathbf{( 6 5 . 6 )}$ & $\mathbf{( 6 1 . 8 )}$ & $\mathbf{( 6 3 . 8 )}$ & $\mathbf{( 7 5 . 0 )}$ & $\mathbf{( 6 9 . 2 )}$ & $\mathbf{( 6 4 . 0 )}$ & $\mathbf{( 6 6 . 0 )}$ \\
\hline
\end{tabular}

${ }^{a}$ GW, Gangwon-do; GG, Gyeonggi-do; GN, Gyeungsangnam-do; GB, Gyeungsangbuk-do; JN, Jeollanam-do; JB, Jeollabuk-do; CN, Chungcheongnam-do; CB, Chungcheongbuk-do.

${ }^{\mathrm{b}}$ Antibody titers equal to or more than 8 were considered as positive.

Korea, veterinary authorities have performed serosurveillance of JEV using approximately 9,000 pig sera annually since 2001. Based on these serologic surveys, preventive measures have been taken to block transmission of the disease. Wild boars acting as amplifiers of JEV are distributed throughout Korea, and their numbers have recently increased (8).

Various serological assays, such as the hemagglutination inhibition (HI) test, plaque reduction neutralization test (PRNT), and enzyme-linked immunosorbent assay (ELISA) have been used to detect specific antibodies against JEV (9 11). The HI test has been considered to be the standard method for detecting JEV antibodies, but cross-reaction between JEV and WNV remains problematic. PRNT has also been determined by plaque reduction, which may be subjective due to observer bias and is not suitable for large-scale seroepidemiological study $(10,12)$. The ELISA provides rapid results, but is expensive for large numbers of samples and provides only positive and negative results. Wild boars are considered to be an important factor in the circulating cycle of birds-swine-mosquitoes and must be considered in the control of swine disease. However, a nationwide serosurvey of JEV in Korean wild boars has not been investigated. In this study, antibodies against JEV in wild boars using a virus neutralizing assay were examined to clarify the JEV infection risk in Korea.

Employed hunters collected a total of 288 blood samples from the jugular vein of wild boars in eight provinces. The blood samples were taken for the purpose of serosurveillance against classical swine fever virus (CSFV) between November and December 2011. The study on serosurveillance against CSFV in wild boars had been approved by the Animal Ethics Committee of our institute
(Animal Ethics number: QIA-AEC-2011-045). Clotted blood samples were separated by centrifugation $(3,000 \times g$, $10 \mathrm{~min}$ ), and the sera were stored at $-20^{\circ} \mathrm{C}$ until use. The KV1899 (genotype 1) strain of JEV was used as the antigen for the virus neutralization (VN) test, which was isolated from Korean pig blood in 1999 and was passaged nine times in Vero cells after isolation (11). The VN tests for JEV were performed in 96-well, flat-bottomed, cell culture microtiter plates using Vero cells. Fifty microliters of wild boar sera and $50 \mu \mathrm{l}$ of medium were added and serially diluted two-fold in $50 \mu \mathrm{l}$ of $\alpha$-MEM (Invitrogen, Grand Island, NY, USA). The diluted samples were then mixed with an equal volume of 100 to 200 TCID $_{50}$ of KV1899 strain and incubated at $37^{\circ} \mathrm{C}$ for $1 \mathrm{~h}$. A total of $100 \mu \mathrm{l}$ of Vero cells was then added to each well at a concentration of $2 \times 10^{4}$ cells in $\alpha$-MEM containing $10 \%$ fetal bovine serum. The microplates were incubated for 5 days at $37^{\circ} \mathrm{C}$ in a $5 \% \mathrm{CO}_{2}$ incubator and examined by microscopy (Nikon, Tokyo, Japan). The VN titer was expressed as the reciprocal of the highest serum dilution completely inhibiting cytopathic effects in the wells. The serum dilution ranged from $1: 2$ to $1: 256$, and antibody titers of $\geq 1: 8$ were considered positive (13). A chi-square test was used to analyze differences in serosurveillance by region. A $p$-value of < 0.05 was deemed to indicate statistical significance (14).

The JEV serosurveillance results in wild boars are shown in Table 1 and Fig. 1. The overall incidence of JEV was $66.0 \%$ in 288 serum samples. In detail, the regional incidences were $81.8 \%$ (45/55) in Gyeonggi-do, 75.0\% (3/4) in Jeollabuk-do, 69.2\% (27/39) in Chungcheongnam-do, $65.6 \%(21 / 32)$ in Gyeungsangnam-do, 64.0\% (16/25) in Chungcheongbuk-do, 63.8\% (30/47) in Jeollanam-do, 61.8\% 


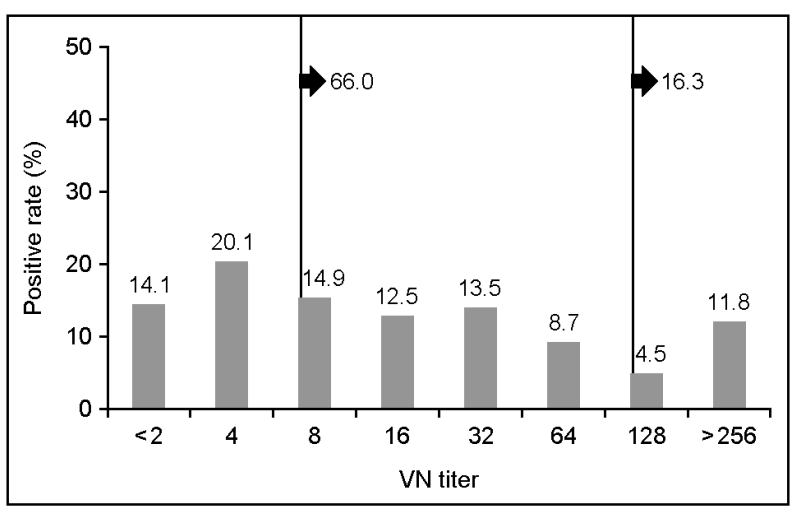

Figure 1. Frequency of distribution of VN titer in 288 serum samples collected from wild boars in Korea.

(21/34) in Gyeungsangbuk-do, and $51.9 \%(27 / 52)$ in Gangwon-do (Table 1). However, there were no significant differences in JEV seroprevalence among provinces $(p>$ 0.05 ). The incidence of wild boars showing a VN titer of $>$ $1: 8$ and $>1: 128$ was $66.0 \%$ and $16.3 \%$, respectively. Of the wild boars with a $\mathrm{VN}$ titer, the most frequent $\mathrm{VN}$ tier was 1:4 (20.1\%) (Fig. 1).

Global warming can increase the incidence of arboviral disease, and vector-borne disease caused by JEV, WNV, and TBEV may be easily introduced into new territories (15). Only one single serotype of JEV has been identified, and both analysis of nucleotide sequence based on the viral premembrane region and phylogenetic analysis of the viral envelope "E" gene have divided JEV strains into four and five distinct genotypes, respectively $(16,17)$. Since 1993, the isolated JEVs in Korea have been classified into genotype 1 (18). Although arthropod-borne viral agents, including JEV, are mainly transmitted through vectors such as Culex spp., a nationwide serosurvey of JEV in wild boars has not been investigated. Thus, we conducted a nationwide survey of JEV antibodies in wild boars. In this study, the sera of wild boars that were collected as part of an annual surveillance program for CSFV were tested for the presence of JEV antibodies. The overall incidence rate of antibodies against JEV was $66.0 \%$. This VN test result suggests that most of the wild boars had been exposed to JEV.

Several researchers reported that wild boars in Japan had
VN antibody levels ranging from 52.2 to $86.7 \%$ (19 21). Because wild boars are closely related to domestic pigs genetically, they are considered to play a similar role in amplifying JEV. According to the regional distribution of JEV antibodies in Table 1, wild boars captured from Gyeonggi-do showed the highest incidence rate (81.8\%). This result may explain the increasing JEV infection rate in Gyeonggi-do, where a high JEV incidence rate was shown in humans between 2007 and 2010 (5). In addition, 16.3\% of wild boars had a VN titer of $>1: 128$. Sugiura et al. (21) reported that horses experimentally inoculated with JEV had high titers within 3 weeks after inoculation. Therefore, wild boars with a titer of $\geq 1: 128$ may have been exposed to JEV very recently. Several positive JEV serosurveillance results have been reported in several species of animals in Korea, such as swine, goats, horses, and cattle (22). Goats are recommended as good sentinel animals for serological monitoring of JEV infection because they are not currently vaccinated in Korea. Like goats, wild boars cannot be immunized with a JEV vaccine, but they are easily exposed to several types of vectors. The high incidence of JEV in Korean wild boars indicates that JEV circulates among birds, swine, and mosquitoes.

In conclusion, the results of the present study indicate that JEV infection is prevalent in Korean wild boars and that these animals might play a key role in the natural transmission cycle of JEV. Further studies involving RTPCR and virus isolation are needed to verify JEV in wild boars.

\section{REFERENCES}

1) Campbell GL, Hills SL, Fischer M, Jacobson JA, Hoke $\mathrm{CH}$, Hombach JM, et al. Estimated global incidence of Japanese encephalitis: a systematic review. Bull World Health Organ 2011;89:766-74.

2) Fischer M, Hills S, Staples E, Johnson B, Yaich M, Solomon T. Japanese encephalitis prevention and control: advances, challenges, and new initiatives. In: Scheld WM, Hammer SM, Hughes JM, editors. Emerging infections 8 Washington: ASM Press; 2008. 
p. 93-124.

3) Duong V, Sorn S, Holl D, Rani M, Deubel V, Buchy P. Evidence of Japanese encephalitis virus infections in swine populations in 8 provinces of Cambodia: implications for national Japanese encephalitis vaccination policy. Acta Trop 2011;120:146-50.

4) Nitatpattana N, Le Flohic G, Thongchai P, Nakgoi K, Palaboodeewat S, Khin M, et al. Elevated Japanese encephalitis virus activity monitored by domestic sentinel piglets in Thailand. Vector Borne Zoonotic Dis 2011;11:391-4.

5) Yang DK, Oh YI, Kim HR, Lee YJ, Moon OK, Yoon H, et al. Serosurveillance for Japanese encephalitis virus in wild birds captured in Korea. J Vet Sci 2011;12:373 -7 .

6) Cao QS, Li XM, Zhu QY, Wang DD, Chen HC, Qian P. Isolation and molecular characterization of genotype 1 Japanese encephalitis virus, SX09S-01, from pigs in China. Virol J 2011;8:472.

7) Gulati BR, Singha H, Singh BK, Virmani N, Kumar S, Singh RK. Isolation and genetic characterization of Japanese encephalitis virus from equines in India. J Vet Sci 2012;13:111-8.

8) Seo SW, Sunwoo SY, Hyun BH, Lyoo YS. Detection of antibodies against classical swine fever virus in fecal samples from wild boar. Vet Microbiol 2012 (in press).

9) Sugiyama I, Shimizu E, Nogami S, Suzuki K, Miura Y, Sentsui H. Serological survey of arthropod-borne viruses among wild boars in Japan. J Vet Med Sci 2009;71: 1059-61

10) Ting SH, See E, Tan HC, Lee MA, Ooi EE. Development of a simplified assay for the detection of neutralizing antibodies to Japanese encephalitis virus. J Virol Methods 2001;93:43-7.

11) Watanabe K, Hirokawa C, Kon M, Tamura T, Nishikawa M. Estimation of focus reduction neutralization test for measurement of neutralizing antibody titer against Japanese encephalitis virus. Jpn J Infect Dis 2008;61: 424-5.

12) Yang DK, Kim BH, Kweon $\mathrm{CH}$, Kwon JH, Lim SI, Han HR. Biophysical characterization of Japanese encephalitis virus (KV1899) isolated from pigs in Korea. J Vet Sci 2004;5:125-30.

13) Boadella M, Díez-Delgado I, Gutiérrez-Guzmán AV, Höfle U, Gortázar C. Do wild ungulates allow improved monitoring of flavivirus circulation in Spain? Vector Borne Zoonotic Dis 2012;12:490-5.

14) Hirota J, Nishi H, Matsuda H, Tsunemitsu H, Shimiz S. Cross-reactivity of Japanese encephalitis virus-vaccinated horse sera in serodiagnosis of West Nile virus. J Vet Med Sci 2010;72:369-72.

15) Mackenzie JS, Gubler DJ, Petersen LR. Emerging flaviviruses: the spread and resurgence of Japanese encephalitis, West Nile and dengue viruses. Nat Med 2004;10:S98-109.

16) Chen WR, Tesh RB, Rico-Hesse R. Genetic variation of Japanese encephalitis virus in nature. J Gen Virol 1990;71:2915-22.

17) Morita K. Molecular epidemiology of Japanese encephalitis in East Asia. Vaccine 2009;27:7131-2.

18) Yun SM, Cho JE, Ju YR, Kim SY, Ryou J, Han MG, et al. Molecular epidemiology of Japanese encephalitis virus circulating in South Korea, 1983-2005. Virol J 2010;7:127.

19) Nidaira M, Taira K, Itokazu K, Kudaka J, Nakamura M, Ohno A, et al. Survey of the antibody against japanese encephalitis virus in Ryukyu wild boars (Sus scrofa riukiuanus) in Okinawa, Japan. Jpn J Infect Dis 2007; 60:309-11.

20) Ohno Y, Sato H, Suzuki K, Yokoyama M, Uni S, Shibasaki $\mathrm{T}$, et al. Detection of antibodies against Japanese encephalitis virus in raccoons, raccoon dogs and wild boars in Japan. J Vet Med Sci 2009;71:1035 $-9$.

21) Sugiura T, Shimada K. Seroepizootiological survey of Japanese encephalitis virus and Getah virus in regional horse race tracks from 1991 to 1997 in Japan. Vet Med Sci 1999; 61: 877-81.

22) Lim SI, Kweon CH, Tark DS, Kim SH, Yang DK. Serosurvey on Aino, Akabane, Chuzan, bovine ephemeral fever and Japanese encephalitis virus of cattle and swine in Korea. J Vet Sci 2007;8:45-9. 